\title{
Reduced Overlap Frontier-based Exploration with Two Cooperating Mobile Robots
}

\author{
Mohammad Al khawaldah \\ Salvatore Livatino \\ David C. Lee \\ [M.M.D.Al-Khawaldah, S.Livatino, D.C.Lee]@herts.ac.uk \\ Department of Engineering and Technology \\ University of Hertfordshire, UK
}

\begin{abstract}
In this paper, a frontier-based exploration is used with a couple of cooperating mobile robots to explore unknown environments. The aim is to decrease the overlap between the robots to minimize the exploration time. The proposed exploration algorithm is based on a bidding function in which a new special parameter was introduced to decrease the overlap between the robots. A large number of experiments have been conducted to analyze the importance of the weight parameters included in the proposed technique. As a result of these experiments, the weight values can be chosen to guarantee short exploration time. The new algorithm has been assessed with different environments with different shapes and different numbers of obstacles. Lastly, the results of our algorithm were compared with the results in the literature. The new technique led to promising results.
\end{abstract}

\section{INTRODUCTION}

The exploration is an important issue in the field of mobile robots. In some applications robots depend on a map to perform their tasks. For instance, robots need the map to find the shortest path between two points to move things between these two points. In other applications the aim is the exploration itself. Such applications may include searching, rescuing and planetary exploration.

Multi-robot systems have the ability to complete their tasks faster than a one robot. In addition, employing more than one robot may increase the redundancy and compensate for robot sensors uncertainty. However, there are some challenges should be addressed in multi-robot systems. The main challenge is coordination between the robots. For example, in an exploration task robots should coordinate their actions to reduce as much as possible the possibility that two different robots explore the same area. Communication among the robots is another problem that should be considered. Good coordination requires reliable communication $[1,2]$.

In [3] Sheng et al provided a fully distributed bidding algorithm for the coordination that considers the limited communication range, and introduces a nearness measure in the bidding algorithm that keeps the robots together. A 2D occupancy grid is used to represent the environment to be explored. A group of robots start from initial positions which are close to each other, and the relative positions are known to all robots. Robots try to explore the area individually with the maximum exploration information and with minimum co\$778-1-4244-6392-3/10/\$26.00 (C) 2010 IEEE
In [4-5] Vazquez, Malcolm and Rocha et al. proposed a behavior-based exploration with multi-robot system. In these techniques the exploration algorithms are implemented in environments represented by means of a global probabilistic grid map. Frontier cells (frontier cell is any free cell for which at least one of its neighboring cells is unexplored) are evaluated according to the estimated cost and the utility of the information. The costs are computed depending on the targetfrontier-cell distance to the robot. And the utility depends on the size of the nearby unexplored area and is equal to the number of unexplored cells existing inside the circumference of the robot sensor range. The utility expected by a robot considering moving to a particular frontier is decreased if there are any robots near that destination. The cell of maximum difference [Utility - Cost] wins the bidding and the robot starts moving towards it.

In all of the previously mentioned published works, nobody optimize choosing the weight (i.e. to investigate the importance) for each of the "Utility" and the "Cost" used in the bidding functions of the exploration algorithms. Furthermore, in these papers, the proposed techniques have not been tested with different environments and different obstacles number.

In this paper a new exploration algorithm is proposed, in which the robots work in pairs and coordinate their actions. Our idea depends on selecting a frontier target cell to increase the efficiency of the exploration. The proposed technique is an extension of the algorithms described in [1, 3-6]. The new technique tries to decrease the overlap between the robots as much as possible. To further improve the performance we also concentrated on finding the best combinations of weight parameters in the frontier-based algorithm used. The new exploration algorithms were tested with different environment sizes, different obstacle distributions and different obstacles numbers. Eventually, we compared the results of our exploration algorithm with the results of one of the known exploration algorithms in the literature [3].

\section{EXPLORATION METHOD}

All of our experiments were conducted using the simulation software - Netlogo [7] which is well known in the literature and employed in many published research works [810]. Netlog enables the computer-based investigation of the $285 x$ ploration process by a number of agents in an occupancygrid-based environment. In Netlog, the environment is 
simulated as an m-by-n grid of square cells. Each cell has information about itself stored in variables. With Netlog, the same experiment can be repeated and results are stored in an Excel file for further analysis.

\section{A. Experiments Assumptions}

All of the experiments presented in this paper use one team of two robots. The map is represented as an m-by-n grid of square cells, each cell of which is allocated a code to represent its occupancy status.

Each robot is equipped with a $360^{\circ}$ sensor, which can detect the occupancy status of all its eight neighbors. This process is known as "scanning". Each robot knows exactly its own position and the position of its partner and they move between the centers of cells. Each robot requires an equal amount of time (a single step in Netlogo) to perform a $360^{\circ}$ scan and move to a neighboring cell. Furthermore, robots can access a shared map of the environment which is updated in every step of the simulation. The communication between the robots is always on, and error free. Finally, the environment edges are treated as occupied cells.

\section{B. Exploration Methodology}

During the process of exploration, each cell of the map is assigned one of the states as shown in table 1 . Table 1 also shows the color code used to identify each state.

Table 1 Cells states and color codes

\begin{tabular}{|c|c|c|}
\hline $\begin{array}{c}\text { Patch } \\
\text { Code }\end{array}$ & Meaning & $\begin{array}{c}\text { Patch Displayed } \\
\text { Colour }\end{array}$ \\
\hline F & "Fresh" No Idea Yet & Gray \\
\hline S & "Free" by Scanning & Brown \\
\hline O & "Occupied" by Scanning & Orange \\
\hline
\end{tabular}

At the beginning of each exploration, all of the cells in the environment are assigned as "F". When a robot visits a cell, all of its free neighbors are assigned to be "S" by scanning as shown in Fig.1. If a robot scans a cell and discovers that there is an obstacle in that cell, this cell is assigned to be an "O" cell as shown in Fig.2. The exploration process is completed when all the cells are explored (free or occupied) as shown in Fig.3.

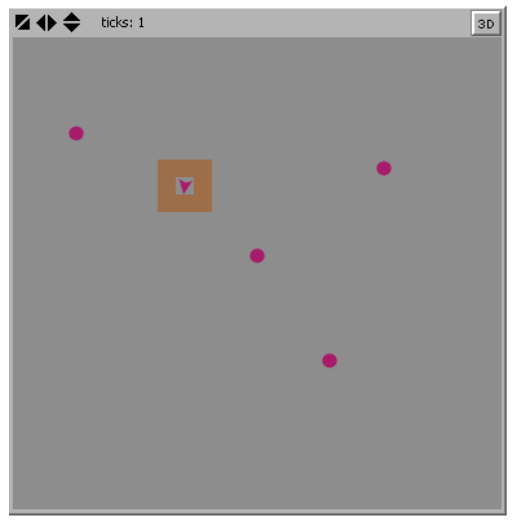

Fig. 1 The environment with one robot after one step of exploration. no any obstacle is near the robot

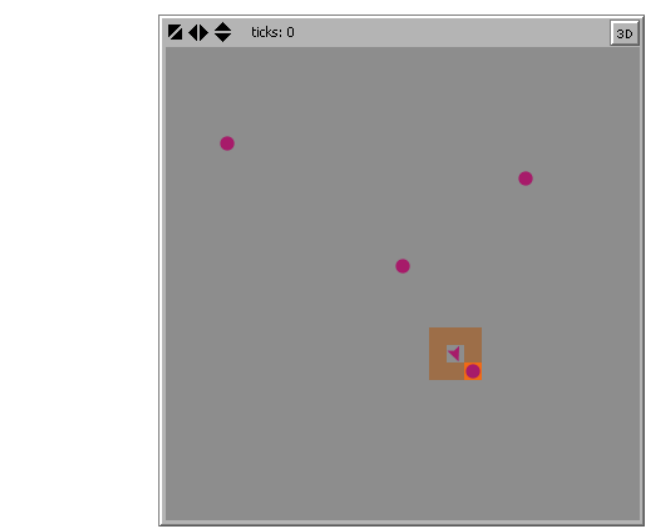

Fig. 2 The environment with one robot after one step of exploration. An obstacle is near the robot

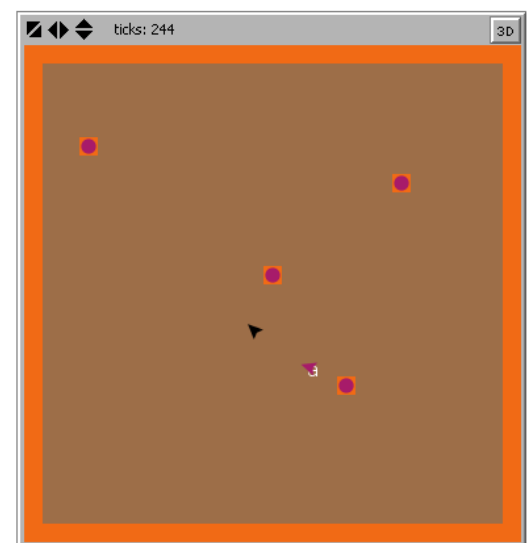

Fig. 3 completed map with two robots

\section{EXPERIMENTATIONS}

A large number of published works in multi-robot exploration depends on the use of "frontier cells" e.g. [1, 3-6, 11]. A frontier cell is any free cell for which at least one of its neighboring cells is unexplored. When a robot is directed to such a cell, it is expected that it will gain information about the unexplored area when it arrives. Because a map may contain several unexplored areas, the challenge arises of how to plan the exploration mission by choosing the most appropriate frontier cells. In $[1,3-6,11]$ the utility of the target frontier cell is computed. The utility of a target cell is the number of unexplored cells which can be scanned from that target cell. Then the cost of reaching that target cell is computed. The cost is a function of the target cell distance. Finally, the target cell with the maximum [Utility - Cost] value wins and the robot starts moving towards its target. This is the general algorithm that guides the exploration in the above mentioned published works.

Apart from the above mentioned two factors - cost and utility, the distance of the other robot from the cell is also considered when computing the bidding value for a frontier cell. This third factor introduces potential benefit of keeping the two robots apart. Each robot computes the bidding value $B_{i}$ for each frontier cell in its shared map based on the bidding function represented in equation (1). The relative importance 2860 each term in the formula is adjusted by a weight value. The most appropriate weight values have been studied and suggestions how they can be chosen are made later in this paper. 


$$
B_{i}=W_{n} N_{u}+W_{p} D_{p}-W_{c} D_{r}
$$

where

$B_{i}$ : The bidding value for the frontier cell $i$ (the target cell).

$D_{r}$ : The distance from the robot to the target cell.

$D_{p}$ : The distance from other robot (the partner) to the target cell.

$N_{u}$ : The number of unknown neighbors for the target cell.

$W_{n}$ (weight neighbors), $W_{p}$ (weight partner) and $W_{c}$ (weight cost) are the weight factors for $\mathrm{N}_{\mathrm{u}}, \mathrm{D}_{\mathrm{p}}$ and $\mathrm{D}_{\mathrm{r}}$, and respectively.

The exploration proceeds as follows:

1. Each robot scans the eight neighboring cells around it and adds the new information (the scan information) to the shared map which is available to both robots.

2. Then each robot computes the bidding function according to equation (1) for all of its frontier cells in the shared map. Each robot has its own bidding function values for the frontier cells in the shared map, computed from its own point of view. A cell can be a frontier cell to more than a robot. The same cell may yield different bidding value to different robots.

3. Each robot chooses among its frontier cells the one with the maximum bidding function value $\max \left\{\mathrm{B}_{\mathrm{i}}\right\}$ and starts moving towards it.

The two robots explore at the same time, and make their decisions on where to go at the next step based on the same shared map. Equation (1) shows that we are trying to guide each robot to a cell with a large number of unexplored neighboring cells, far away from its partner, and close to its current position.

The weights $W_{n}, W_{p}$ and $W_{c}$ were introduced, to investigate the relative importance of each of the three parameters $N_{u}, D_{p}$ and $\mathrm{D}_{\mathrm{r}} . W_{n}$ in our technique represents the weight of the utility expected from going to the frontier cell. In other words, it reflects the importance of the size of the region that will be explored when the robot visits that frontier cell. The weight $W_{p}$ is introduced to reflect the importance of keeping the robots away from each other. Keeping the robots away from each other may, sometimes, make each robot explore on its own a different and relatively large portion of the environment completely. This appears to be better than making each robot explore small areas in different places in the environment, as there is a need to come back again to explore the unexplored spots between the explored areas. In addition, keeping the robots away from each other reduces the probability of overlap. $W_{c}$ represents weight for the cost function. For distant frontier cells the $W_{c} D_{r}$ term reduces their bidding function value, because the value of this term is subtracted from the bidding function, and hence reduces their probability to win the bidding.

For a particular set of $W_{n}, W_{p}$ and $W_{c}$ in a given environment, a problem appears in which a robot starts to oscillate between two cells. The robot moves one step towards its target (called target number one) then it recalculates the bidding function and moves towards another cell (called target number two), and after that it calculates the bidding function again and moves towards the same previous target (target number one) and so on. In such a case, the robot oscillates between the two targets. This will not cause a dead loop as the oscillation will cease after approximately one to three steps. The oscillation disappears because the bidding function for a robot also depends on the movement of its partner or, more precisely, the distance between the frontier cell (the target) and its partner, which changes its position at each step independently. So, the winning target cell will change with the movement of the other robot. However, the oscillation can become a serious problem when both robots start to oscillate together. In such cases, the oscillation continues forever and becomes a dead loop. This problem has been solved by detecting the oscillation and then making one or both of the oscillating robots jump to a randomly chosen free neighboring cell.

\section{A Experiment Design}

The aim now is to find the appropriate combinations of the weight values that lead to relatively short exploration times. The idea is to execute many exploration experiments. Each experiment is conducted with different set of weight values $\left(W_{n}, W_{p}\right.$ and $\left.W_{c}\right)$. After that, the way in which these different weight sets affect the exploration time is investigated and analyzed. We started with an environment size of 25 -by-25 cells. This environment size requires a relatively short but reasonable exploration time with which we can perform a large number of experiments. The results of these experiments will provide us with an idea about how the exploration time is affected with different weight values. In particular, we will focus on the weights that lead to comparatively short exploration time ("good weights").

Then the same procedure is repeated for the same environment but with different numbers of obstacles $(0,10$, 20, 30 and 40 obstacles). In particular, we try to see whether the "good weights" are affected by the number of obstacles or not.

We also thought that it would be useful to test our algorithm with a different environment size to see how the good weight values vary with the size of the environment. An environment of 31 -by-31 cells was chosen. The time required to explore this environment will be relatively longer than the ones of size 25 -by-25 cell but still reasonable.

Then the same procedure is repeated with a different environment shape (20-by-25 cells) in order to see if the "good weights" are affected by different environment shapes.

The effectiveness of each exploration is evaluated by counting the number of steps until the environment has been completely explored, and each cell has been identified as free or occupied.

To see the importance of the three weight values $W_{n}, W_{p}$ and $W_{c}$, one thousand experiments for each of the above mentioned environments have been conducted. Positions of the obstacles are fixed in all of the one thousand experiments. Each experiment was conducted with a different set of weight values. For example, in the first experiment $W_{n}=1, W_{p}=1$, 
and $W_{c}=1$, in the second experiment $W_{n}=2, W_{p}=1$, and $W_{c}=1$, and in the thousandth experiment $W_{n}=10, W_{p}=10$, and $W_{c}=10$.

\section{B Results}

We are interested only in the sets of $W_{n}, W_{p}$ and $W_{c}$ values that lead to short exploration time. We focus on the sets of $W_{n}, W_{p}$ and $W_{c}$ that generate the shortest 10 percent of exploration times. For example, after trying one thousand different sets of $W_{n}, W_{p}$ and $W_{c}$ values, if the minimum exploration time obtained is 100 and the maximum (worst case) is 400 , then the exploration time ranges from 100 to 400. We concentrate on lowest 10 percent of the range, that means we only accept the sets of $W_{n}, W_{p}$ and $W_{c}$ that can lead to an exploration time of not more than 130 . To study the relative values of $W_{n}, W_{p}$ and $W_{c}$ and their effects on the exploration time we put all the data from the one thousand experiments into an Excel sheet. Each row contains $W_{n}, W_{p}$ and $W_{c}$ values in addition to the corresponding steps number for that experiment. For two of the environments mentioned above (environments of ten and twenty obstacles with 25-by25 cells), eight thousand experiments were repeated with $W_{n}$, $W_{p}$ and $W_{c}$ varying from 1 to 20 . No better result was obtained. This indicates that no need for the weight values to go beyond ten.

It should be noted that in (1) $W_{n}, W_{p}$ and $W_{c}$ represent relative not absolute importance. For example, choosing $W_{n}=$ $1, W_{p}=2$ and $W_{c}=3$ is same as choosing $W_{n}=2, W_{p}=4$ and $W_{c}=6$, and they lead to the same set of movements of robots and hence the same total number of steps (exploration time). Considering this, $W_{c}$ is fixed at 1 in all our next experiments while $W_{p} \& W_{n}$ are varied from 0 to 10 in steps of 0.1 independently. This means that for each of the above mentioned environments ten thousand experiments have been conducted again. Figs.4-8 show the results of these experiments. The black squares show the combinations of $W_{p}$ $\& W_{n}$ that have resulted in the best 10 percent of the exploration time.

Fig. 4 shows the results for an 25-by-25 environment with no obstacle where the black squares are concentrated between $W_{p}=0-4 \& W_{n}=0-4$, meaning that high values of $\mathrm{W}_{\mathrm{p}} \&$ $\mathrm{W}_{\mathrm{n}}$ leads to long exploration time. The same can be observed in Fig.s 5-7, where neither of $\mathrm{W}_{\mathrm{p}} \& \mathrm{~W}_{\mathrm{n}}$ should ever go beyond 6 or 7 . This observation confirms the necessity of introducing the cost of the robot moving to the target frontier cell (i.e. the term associated with $W_{c}$ ) into (1).

In Figs.4-8, the black squares scatter around the line of slope equal to 1 . This indicates that the terms associated with $\mathrm{W}_{\mathrm{p}} \& \mathrm{~W}_{\mathrm{n}}$ are equally important and should both be considered when calculating the bidding function. Fig.8 shows the best combinations of weight values for 15 obstacles but with an environment area equal to one and half times that of the previous environments (31-by31 cells). This bigger environment has been introduced to see the effect of the size of the environment on the weight values. Again, the black squares scatter around the line of slop 1 .

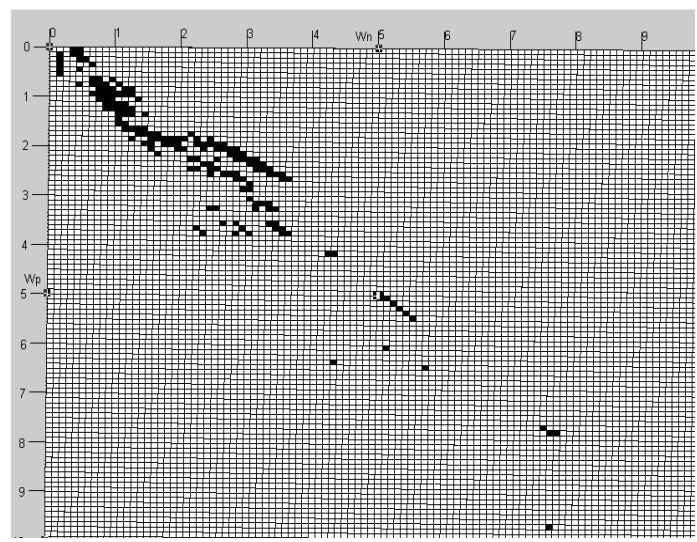

Fig.4 The weight values with no obstacles.

The black squares are the weights of the minimum exploration time experiments in this environment when $W_{c}=1$.

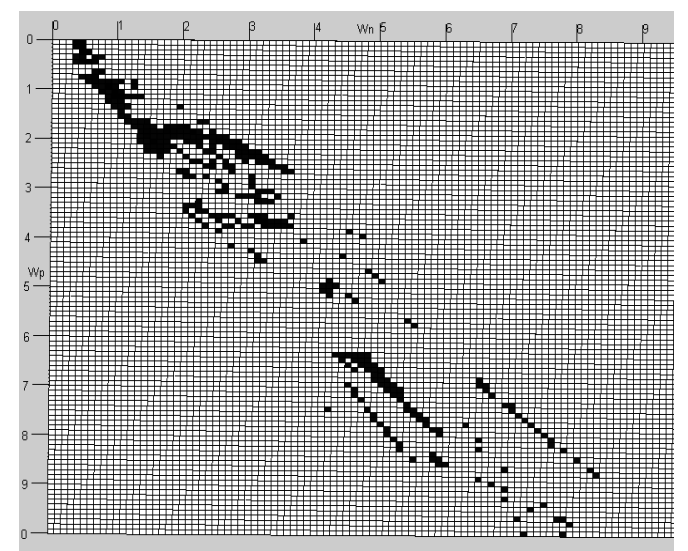

Fig. 5 The best weight values with 10 scattered obstacles. The black squares are the weights of the minimum exploration time experiments in this environment when $W_{c}=1$.

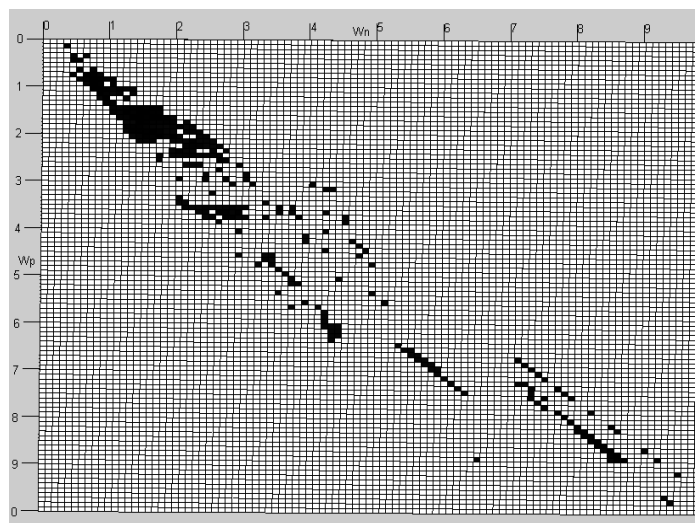

Fig. 6 The best weight values with 20 scattered

obstacles. The black squares are the weights of the minimum exploration time experiments in this environment when $W_{c}=1$. 


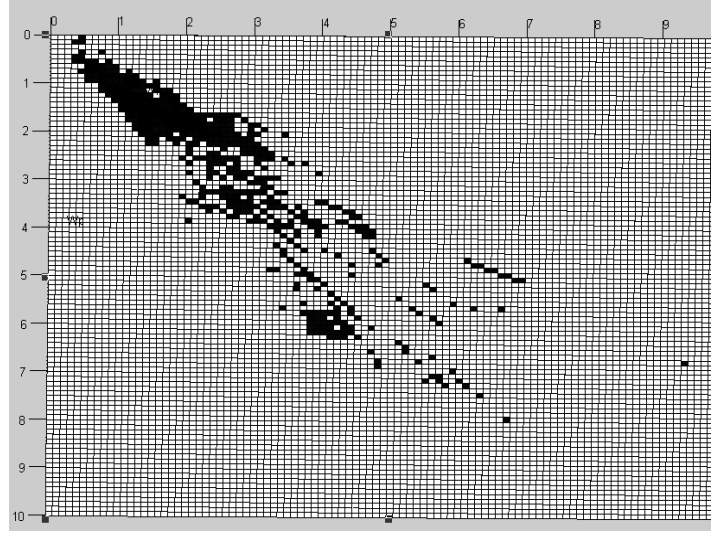

Fig. 7 The best weight values with 40 scattered obstacles. The black squares are the weights of the minimum exploration time experiments in this environment when $W_{c}=1$.

Approximately the same results have been obtained with the rectangle-shaped (20-by-25 cells) environment. Based on this result, it can be concluded that changing the environment size does not change the best weight values.

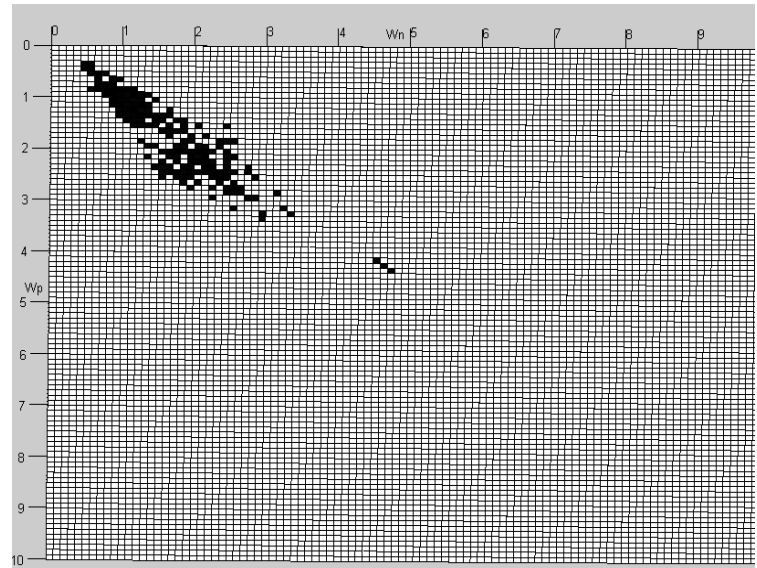

Fig. 8 The best weight values with 15 scattered obstacles. The environment is 31 by 31 cells ( 961 cells size). The black squares are the weights of the minimum exploration time experiments when $W_{c}=1$.

Based on these results, we have come to the conclusion that changing the obstacles number, environment size and environment shape has a very slight influence on the best weight values. It is recommended to choose weight values ( $W_{n}$ and $W_{p}$ ) such that they lie on or close to a line of slop equal 1. And based on the figures obtained, we recommend the same value for both $W_{n}$ and $W_{p}$ and to be any value between 0.5 and 3.0 and for sure with $W_{c}=1$. Furthermore, we tested some weight combinations that lie very close to the line of slop equal 1 but not in the area of the black squares. That means they are not in the best ten percent exploration times. It was noticed that these weight combinations lead to exploration time that is very close to the best ten percent ones.

We can suggest many weight combinations to guarantee a short exploration time (i.e. within or very close to the best 10 percent). For example, we can suggest the weight values to be as follows $W_{p}=2, W_{n}=2$ and $W_{c}=1$. This combination leads to an exploration time around 125 steps in all of the above mentioned environments of size 25 -by- 25 cells. This exploration time is one of the best ones in these experiments.

\section{RESULTS ANALYSIS}

In this section, the algorithm proposed in this paper is tested across a range of environments. All of the environments are 25-by-25 cells. The results are shown in Table 2.

In Table 2 we also compare our work with the exploration algorithm in [4]. The algorithm in [3] is much related to ours but its bidding function is slightly different. In this algorithm the robots choose their next frontier target cell according to the following equation:

$$
g_{i}=w_{1} I_{i}-w_{2} D_{i}+w_{3} \lambda_{i}
$$

where:

$I_{i}$ : The information gain for the frontier cell $i$ (the number of unexplored cells within the robot sensor range but, at the same time, not in the range of other robots or target cells for other robots)

$D_{i}$ : The shortest travelling distance to the frontier cell $i$.

$\lambda_{i:}$ Is the nearness measure.

$w_{1}, w_{2}$, and $w_{3}$ are the weights for these three parameters and respectively.

The nearness measure is included in this equation to keep the robots close to each other to guarantee the communication amongst them. But in our simulation it is assumed that the robots operate within their communication range, we just focus on the exploration algorithms. In practice, when the entire area to be explored is larger than the communication range, we can divide the area into smaller blocks and explore them block by block. So the robots can share their maps in each step. Therefore, the nearness measure $\left(\lambda_{i}\right)$ in (2) is ignored, by setting $w_{3}$ to zero, when we compare the results of our technique (which is based on (1)) with this technique based on (2). $w_{1}$ and $w_{2}$ are set to 1 as Sheng suggests in his simulation in [3].

Table 2 shows comparisons between the experiments' results for both of these exploration techniques. The weights used in these experiments are $W_{p}=2, W_{n}=2$ and $W_{c}=1$ as suggested earlier. Fig.10 shows how the exploration time varies with number of scattered obstacles.

Table 2 Comparisons between the experiments' results for the exploration techniques presented in this paper. All results are averages across 10 experiments for each technique in each environment

\begin{tabular}{|l|l|c|}
\hline & Sheng 2006 & Frontier-Bsed \\
\hline No Obstacles & 158.7 & 128 \\
\hline One Obstacle & 155.4 & 127 \\
\hline Two Obstacles & 155.7 & 126.2 \\
\hline Five Obstacles & 155.2 & 124.6 \\
\hline 10 Obstacles & 153.3 & 122.6 \\
\hline 20 Obstacles & 163.3 & 123.3 \\
\hline 30 Obstacles & 158.8 & 122.9 \\
\hline 40 Obstacles & 155.6 & 123.1 \\
\hline One Block & 158.1 & 122.2 \\
\hline Two blocks & 161.1 & 130 \\
\hline Four blocks & 165 & 119.5 \\
\hline Six Blocks & 168 & 135 \\
\hline
\end{tabular}


It can be observed that our proposed frontier-based exploration algorithm, with its recommended weight parameters, is always better than the Sheng et. al algorithm

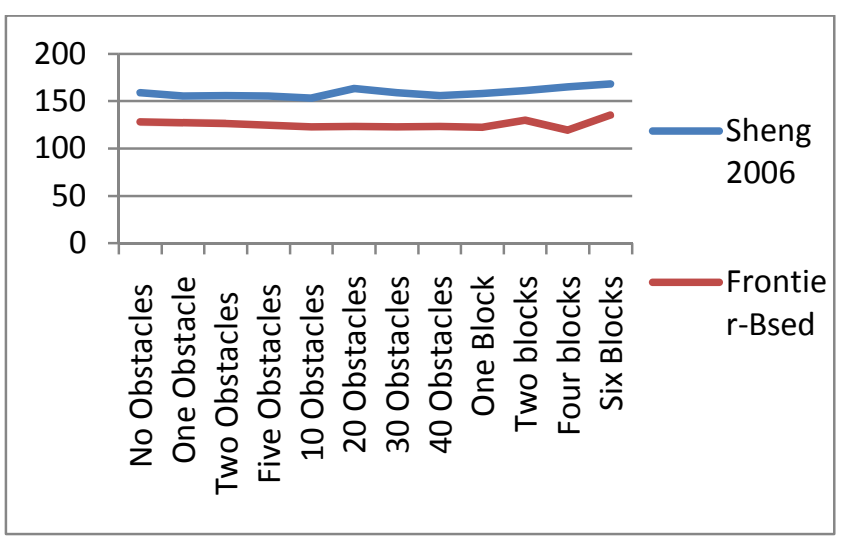

Fig.9 Exploration time (steps) vs. number of scattered obstacles

in terms of the exploration time.The exploration time with frontier-based algorithm seems to be fixed if the environment size is fixed whatever the number of obstacles is. Actually the exploration time decreases slightly with an increase of the obstacles number because the free area to be explored becomes smaller. Finally, changing the scattered obstacles distribution does not affect the exploration time

.To generalize these results we had, new set of experiments included block of obstacles (six occupied cells each). The same procedure is repeated with different numbers of bocks of obstacles. The experiments included two blocks, four blocks and six blocks of obstacles. To have more generalized results, the same previous sets of experiments that included block of obstacles (six occupied cells each) were repeated but with different positions. In each set of experiments, the positions of the blocks of the obstacles are changed to analyze how the appropriate weight varies with changing the blocks' places. The experiments included two blocks, four blocks and six blocks of obstacles. The result of these new experiments sets confirmed that the appropriate weight values which lead to short exploration time are slightly affected by the obstacle distributions (i.e. obstacle shapes and positions). Figure 10 shows an environment with scattered 21 scattered obstacles and Figure 11 shows an environment with four blocks of obstacles.

\section{CONCLUSION}

This paper proposes an exploration algorithm in which a team of two mobile robots are used to perform an exploration task. The algorithm is an extension of the frontier-based algorithms known in the literature. The proposed technique tries to reduce the overlap as much as possible between the robots. Many experiments have been conducted to optimize the weight parameters used in this technique. This algorithm with the suggested (recommended) weight values have been tested with environments with $0,1,2,5,10,20,30$ and 40 obstacles and with different environment shapes and sizes. Then, it was tested with the following block of obstacles (each one is six small obstacles) 1, 2, 4 and 6 blocks. Results with all of these environments have confirmed that the environment characteristics (i.e. shape, size, number and distribution of obstacles) have a slight effect on the optimum weight parameter values used in this algorithm. Our algorithm effectively reduced the exploration time compared to the results in the literature.

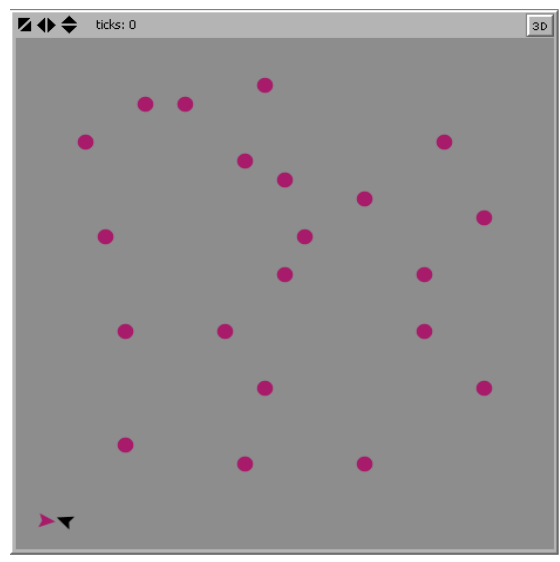

Fig.10 An environment with scattered 21scattered obstacles

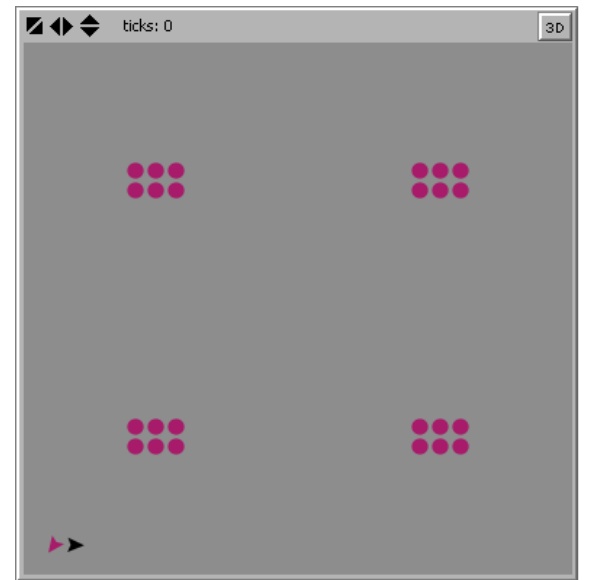

Fig.11 An environment with four blocks of obstacles

\section{EFERENCES}

[1] W. Burgard, M. Moors, C. Stachniss, \& F. Schneider, "Coordinated multi-robot exploration," IEEE Transactions on Robotics. 21(3): p. 376-386, 2005

[2] M. Al-khawaldah, and D. Lee, "Cooperative robot exploration with Line-of-Sight technique," International Conference on Information and Communication Systems: Jordan/Amman. to appear ,2009.

[3] W. Sheng, Q. Yang, J. Tan, \& N. Xi "Distributed multi-robot coordination in area exploration," Robotics and Autonomous Systems, 54(12): p. 945-955, 2006.

[4] Vazquez, J. and C. Malcolm, "Distributed multi-robot exploration maintaining a mobile network" International IEEE conference in intelligent systems, 2004.

[5] R. Rocha, J. Dias, and A. Carvalho, "Cooperative multi-robot systems: A study of vision-based 3-D mapping using information theor," Robotics and Autonomous Systems, 53(3-4): p. 282-311, 2005.

[6] D. Fox, J. Ko, K. Konolige, B. Limketkai, D. Schulz, \& B. Stewart, "Distributed multi-robot exploration and mapping," Proceedings-IEEE, 94(7): p. 1325, 2006.

[7] U. Wilensky, NetLogo. http://ccl.northwestern.edu/netlogo/. Center for Connected Learning and Computer-Based Modeling, Northwestern University. Evanston, IL, 1999.

[8] S. Magg, and R.E.T. Boekhorst, "Pattern formation in homogeneous and heterogeneous swarms: differences between versatile and specialized agents," IEEE Symposium on Artificial Life, 2007.

[9] M. Niazi, and A. Hussain, "Agent-Based Tools for Modeling and Simulation of Self-Organization in Peer-to-Peer, Ad Hoc, and Other Complex Networks," in IEEE Communications Magazine, 2009.

6614] R. Menezes, and H. Bullen, "A study of terrain coverage models," In ACM symposium on applied computing, 2008.

[11] W. Burgard, M. Moors, and F. Schneider, "Collaborative exploration of enknown Environments with teams of mobile robots," lecture Notes In Computer Science, p. 52-70, 2002. 